\title{
Comment to the paper: incidence of mutism, dysarthria, and dysphagia associated with childhood posterior fossa tumor
}

\author{
Charles Teo
}

Received: 8 March 2011 / Accepted: 10 March 2011 /Published online: 1 April 2011

(C) Springer-Verlag 2011

This is an excellent review of post-operative dysphagia, dysphonia, and mutism following surgery for posterior fossa tumors. The authors have done a thorough literature search and precise and comprehensive data collection of their own patients. The findings are not earth shattering but consistent with the current literature. The incidence is much higher than one would expect and arguably would be higher if the study was prospective. I am alarmed and somewhat surprised at the high incidence of dysphagia, dysphonia, and mutism and that all three deficits were consistently present in a high percentage at discharge. My initial conclusion was that the surgeons were probably aggressive and that these were acceptable consequences of radical and curative surgery. Unfortunately the authors report a very high incidence of incomplete resection of mostly benign tumors $(63 \%)$. This is disturbing. One justification of this surgical outcome would be if the study showed better neurological status in those patients in whom tumor was purposefully left behind. As this is not the case, I wonder if sub-total resection may be a negative prognosticator in eventual neurological outcome?

\section{Charlie Tea AM}

www.neuroendoscopy.info
C. Teo $(\bowtie)$

Centre for Minimally Invasive Neurosurgery,

Randwick, NSW, Australia

e-mail: cteo@bigpond.net.au 